\title{
PARAMETERS AND DRIVING FORCE ESTIMATION OF CELL MOTILITY VIA EXPECTATION-MAXIMIZATION (EM) APPROACH
}

\author{
Dejan Milutinović * \\ Applied Mathematics and Statistics Department \\ University of California \\ Santa Cruz, California 95064 \\ Email: dejan@soe.ucsc.edu
}

\author{
Devendra P. Garg \\ Mechanical Engineering and Materials Science Department \\ Duke University \\ Durham, North Carolina, 27708 \\ dpgarg@pratt.duke.edu
}

\begin{abstract}
Motility is an important property of immune system cells. To describe cell motility, we use a continuous stochastic process and estimate its parameters and driving force based on a maximum likelihood approach. In order to improve the convergence of the maximization procedure, we use expectation-maximization (EM) iterations. The iterations include numerical maximization and the Kalman filter. To illustrate the method, we use cell tracks obtained from the intravital video microscopy of a zebrafish embryo.
\end{abstract}

Keywords: Expectation-maximization, Kalman filter estimation, Cell motility, Molecular and cellular bioimaging.

\section{NOMENCLATURE}

$\mathbf{I}_{D \times D}, \mathbf{0}_{D \times D}$ the unity and zero matrices of the dimension $D$.

$t, t_{k}$ the time and the time at the sample point $k$.

$\mathbf{q}, \mathbf{p}$ the position and velocity vectors.

$\mathbf{x}, \mathbf{y}$ the state and measurement vectors.

$\mathbf{f}_{\mathbf{0}}$ the force vector.

$\mathbf{e}_{k} \quad$ the prediction error vector at the sample point $k$.

$\xi(t)$ the Gaussian white noise of the unit intensity.

$\theta(t), \Theta$ the measurement error and the corresponding covariance matrix.

$\mathcal{L}$ the log-likelihood of the data.

$\mathbf{K F}_{\mathbf{f}_{\mathbf{0}}}$ the Kalman filter providing the force vector estimation. $E, E_{\mathbf{f}_{\mathbf{0}} \sim N\left(\hat{\mathbf{f}}_{\mathbf{0}}, \Sigma_{\mathbf{f}_{\mathbf{0}}}\right)}\{\cdot\}$ the expectation operator and the expected value of $\{\cdot\}$ with respect to the probability density function of the force $\mathbf{f}_{\mathbf{0}}$, which is the Gaussian distribution with the mean value $\hat{\mathbf{f}}_{\mathbf{0}}$ and the covariance matrix $\Sigma_{F}$.

\footnotetext{
*Address all correspondence to this author.
}

\section{INTRODUCTION}

Studying biological cells in their physiological environment is important for quantitative understanding of spatio-temporal scale of intra-vital cellular interactions. Recent developments in video microscopy provide information about immune system cells residing in tissues of living organisms, such as zebrafish embryo [1], or mouse [2,3]. This visual information can be used to identify characteristics of cell motility, which is specially important in the case of immune system cells whose functionality is closely related to their ability to move [4].

An effective force driving the immune system cell motility originates from the mechanical forces of the extracellular environment and the active responses of the cell to gradients of chemotactic and haptotactic substances. Currently, there is no microscopy method available for measuring these forces under physiological conditions. Therefore, we can only rely on an effective force estimation from data gathered from intra-vital video microscopy.

The goal of this paper is to present an expectationmaximization (EM) approach [5] for estimating parameters and the driving force of cell motility. This problem falls into a class of the combined parameter and state estimation problems involving a hierarchical identification principle $[6,7]$. Whereas the application of the EM approach in the case of discrete-time models and regularly sampled data has been introduced [8], an EM approach for a time-continuous model and irregularly sampled data has not been discussed yet in the literature. Consequently, the development of an EM approach for the continuous-time stochastic process describing cell motility is the main contribution of this paper. It provides us directly an estimation of the continuoustime stochastic process, without dealing with nonlinear relations between continuous process parameters, discrete-time model pa- 
rameters and irregular intersample intervals.

In general, unknown model variables can be estimated using maximization of the likelihood resulting from the prediction error method [9]. This method has been used both for the parameter estimation of discrete-time stochastic processes [10] and time-continuous stochastic processes with discrete-time measurements $[11,12]$. A straightforward approach for implementing this method is to maximize the likelihood function numerically. However, numerical optimization usually suffers from the convergence problem [12]. Avoiding this problem is the main reason for considering the EM approach presented here.

In the EM approach, we divide the original problem of estimating parameters and forces into two sub-problems: the first being the problem of estimating parameters for a given distribution of the force vector (M-step) and the second being the problem of estimating the force vector for given parameters (E-step). We solve the first problem (M-step) by using numerical optimization and the second (E-step) by utilizing the Kalman filter [13]. The convergence of EM iterations is discussed in [5, 14, 15]. For the convergence, it is not necessary that parameter values computed in the M-step correspond to exact maximum of the likelihood. Therefore, the application of a numerical procedure in the $\mathrm{M}$ step is appropriate.

The paper is organized as follows. In Section 2, we introduce the model for quantifying a cell motility including parameters and the force vector. The model-based likelihood of observed cell trajectory is discussed in Section 3 and the EM iterations are described in Section 4. Section 5 provides an example of estimating cell motility from intravital video of macrophages of zebrafish embryo and conclusions are presented in Section 6.

\section{THE CELL MOTILITY MODEL AND DATA}

The model we introduce here and the data we consider describe the motion of the cell volume center. The model parameters are values $c$ and $\sigma$ and the model is given by the following equation:

$$
\begin{aligned}
\dot{\mathbf{q}}(t) & =\mathbf{p}(t) \\
\dot{\mathbf{p}}(t) & =-c \mathbf{p}(t)+\sigma \xi(t)+\mathbf{f}_{0}
\end{aligned}
$$

in which $\mathbf{q}$ is the cell position, $\mathbf{p}$ is the cell velocity, $\xi(t)$ is the Gaussian white noise of the unit intensity and $\mathbf{f}_{0}$ is the constant force resulting in the cell motility drift. All of these values are vectors of dimension $D$, where $D=2$ and $D=3$ correspond to two- and three-dimensional cell motility models, respectively. It is worth mentioning that this type of double integrator model representing particle dynamics [16-18] is common in representing the motion of agents in a multi-agent system. The right-hand side of (2) is a control of the agent motility, i.e., cell motility. The structure of control and stability analysis has been discussed in [18] and influence of stochasticity term in [19].
To keep the notation simple, we introduce a state vector $\mathbf{x}$, which in our case is the column vector $\mathbf{x}=[\mathbf{q} \mathbf{p}]^{T}$. The cell motility model in the matrix form is

$$
\begin{gathered}
\dot{\mathbf{x}}(t)=\mathbf{A x}(t)+\mathbf{L} \xi(t)+\mathbf{B}, \mathbf{B}=\left[\begin{array}{c}
0 \\
\mathbf{f}_{0}
\end{array}\right] \\
\mathbf{A}=\left[\begin{array}{cc}
\mathbf{0}_{D \times D} & \mathbf{I}_{D \times D} \\
\mathbf{0}_{D \times D} & -c \mathbf{I}_{D \times D}
\end{array}\right], \mathbf{L}=\left[\begin{array}{c}
0 \\
\sigma \mathbf{I}_{D \times D}
\end{array}\right]
\end{gathered}
$$

where I is the identity matrix.

The data obtained from cell motility movies are positions of the cell volume center. These positions are not measurement error free. If the measured cell position at time point $t_{k}$ is $\mathbf{y}_{k}$, then,

$$
\mathbf{y}_{k}=\mathbf{q}\left(t_{k}\right)+\theta\left(t_{k}\right)=\mathbf{C x}\left(t_{k}\right)+\boldsymbol{\theta}\left(t_{k}\right), \quad \mathbf{C}=\left[\mathbf{I}_{D \times D} \mathbf{0}_{D \times D}\right]
$$

where $\theta\left(t_{k}\right)$ is the random error vector assigned to the observed position of the cell volume center. We designate equation (5) as the measurement error model. We assume that the error $\theta$ is a zero-mean-value Gaussian vector variable with covariance $\Theta$. Moreover, we assume that the errors along the coordinate axes are uncorrelated, so that the matrix $\Theta$ is diagonal. Throughout the paper, we assume that the covariance $\Theta$ of measurement errors is known. This is due to the fact that the measurement errors can be easily estimated, or guessed independently of the way cells move.

\section{DATA LIKELIHOOD}

The complete model for the measured positions of the cell volume center is given by equations (3) to (5). Let us assume that at time instant $t_{k}$ the expected value of the state is known, which includes the position and velocity of the cell, $\hat{\mathbf{x}}_{k}$ and covariance matrix $\mathbf{P}_{k}$ of the state vector. Based on this information, we can predict the expected value of the state at the time $t_{k+1}, \overline{\mathbf{x}}\left(t_{k+1} \mid t_{k}\right)$ and its covariance $\Sigma\left(t_{k+1} \mid t_{k}\right)$ by solving the following ordinary differential equations [13] on the interval $\left[t_{k}, t_{k+1}\right]$ :

$$
\begin{aligned}
\dot{\overline{\mathbf{x}}}\left(t \mid t_{k}\right) & =\mathbf{A} \overline{\mathbf{x}}\left(t \mid t_{k}\right)+\mathbf{B}, \quad \overline{\mathbf{x}}\left(t_{k} \mid t_{k}\right)=\hat{\mathbf{x}}_{k} \\
\dot{\Sigma}\left(t \mid t_{k}\right) & =\mathbf{A} \Sigma\left(t \mid t_{k}\right)+\Sigma\left(t \mid t_{k}\right) \mathbf{A}^{T}+\mathbf{L}^{T} \mathbf{L}
\end{aligned}
$$

where $\Sigma\left(t_{k} \mid t_{k}\right)=\mathbf{S}_{k}$ and

$$
\mathbf{S}_{k+1}=\left(\mathbf{I}-\mathbf{K}_{k} \mathbf{C}\right) \Sigma\left(t_{k+1} \mid t_{k}\right)\left(\mathbf{I}-\mathbf{K}_{k} \mathbf{C}\right)^{T}+\mathbf{K}_{k} \Theta \mathbf{K}_{k}^{T}
$$

with $\mathbf{K}_{k}$ computed as,

$$
\mathbf{K}_{k}=\Sigma\left(t_{k+1} \mid t_{k}\right) \mathbf{C}^{T}\left(\mathbf{C} \Sigma\left(t_{k+1} \mid t_{k}\right) \mathbf{C}^{T}+\Theta\right)^{-1}
$$


Taking into account the last expression, equation (8) can be written in a simpler form as,

$$
\mathbf{S}_{k+1}=\left(\mathbf{I}-\mathbf{K}_{k} \mathbf{C}\right) \Sigma\left(t_{k+1} \mid t_{k}\right)
$$

Using these values, we can also predict the expected value of the measured cell position and its covariance at the time point $t_{k+1}$ as

$$
\begin{aligned}
\overline{\mathbf{y}}_{k+1} & =\mathbf{C} \overline{\mathbf{x}}\left(t_{k+1} \mid t_{k}\right) \\
\mathbf{P}_{k+1} & =\mathbf{C S}_{k+1} \mathbf{C}^{T}+\Theta
\end{aligned}
$$

Furthermore, we can combine these predictions (priors), using Bayes' rule, with the information obtained from the measurement $\mathbf{y}_{k+1}$, which is a Gaussian random variable (see equation (5)). Therefore, we can compute posterior estimation of the state $\hat{\mathbf{x}}_{k+1}$ and its covariance at the time point $t_{k+1}$ using the following expression [13]:

$$
\hat{\mathbf{x}}_{k+1}=\overline{\mathbf{x}}\left(t \mid t_{k}\right)+\mathbf{K}_{k}\left(\mathbf{y}_{k+1}-\overline{\mathbf{y}}_{k+1}\right)
$$

Expressions (6) to (9) define the Kalman filter, which is one of the major results of optimal estimation theory for dynamical systems [13]. The Kalman filter computes the optimal state estimation $\hat{\mathbf{x}}_{k+1}$ based on data $\mathbf{y}_{k}, k=0,1, \ldots, k+1$. Filter iterations include the prediction (6)-(12) and update (13) steps. The error between the actual measurements and the predicted value is the prediction error

$$
\mathbf{e}_{k+1}=\mathbf{y}_{k+1}-\overline{\mathbf{y}}_{k+1}
$$

Since we are dealing with the linear stochastic system in which the state and measurement vectors are Gaussian, the prediction error is also Gaussian with the zero-mean-value and covariance matrix $\mathbf{P}_{k+1}$. Thus,

$$
\mathbf{e}_{k+1} \sim \mathcal{N}\left(\mathbf{0}, \mathbf{P}_{k+1}\right)
$$

Moreover, the prediction error is a white noise sequence [13] since

$$
E\left\{\mathbf{e}_{k} \mathbf{e}_{j}\right\}=0, \forall k \neq j
$$

where $E$ denotes the expectation operator.

It has been shown in [20] that one way to evaluate the loglikelihood of data is to use the prediction error and the following relationship:

$$
\mathcal{L}=-\sum_{k=1}^{N} \mathbf{e}_{k} \mathbf{P}_{k}^{-1} \mathbf{e}_{k}+\log \operatorname{det} \mathbf{P}_{k}
$$

In order to compute the likelihood function using the Kalman filter, we need to start with some a priori distribution of the state vector specifying $\hat{\mathbf{x}}_{0}$ and $\mathbf{S}_{0}$. In the applications of the Kalman filter, whenever $\mathbf{x}_{0}$ is unknown, we can use "large" $\mathbf{S}_{0}$; for example, a diagonal matrix with large values along the diagonal terms. For the analysis of cell tracks, the vector $\mathbf{x}_{0}$ and matrix $\mathbf{S}_{0}$ can be initialized as,

$$
\mathbf{x}_{0}=\left[\begin{array}{l}
\mathbf{q}\left(t_{0}\right) \\
\mathbf{p}\left(t_{0}\right)
\end{array}\right]=\left[\begin{array}{c}
\mathbf{y}_{0} \\
\frac{1}{t_{1}-t_{0}}\left(\mathbf{y}_{1}-\mathbf{y}_{0}\right)
\end{array}\right]
$$

$$
\mathbf{S}_{0}=\left[\begin{array}{cc}
\Theta & \mathbf{0}_{D \times D} \\
\mathbf{0}_{D \times D} & \frac{2 \Theta}{\left(t_{1}-t_{0}\right)^{2}}
\end{array}\right]
$$

This is because the position of the cell $\mathbf{p}\left(t_{0}\right)$ at time $t_{0}$ cannot be far from the measured position $\mathbf{y}_{0}$. Similarly, the cell position $\mathbf{q}\left(t_{1}\right)$ at time $t_{1}$ is close to the measured position $\mathbf{y}_{1}$. Due to that, we can estimate roughly the velocity $\mathbf{p}\left(t_{0}\right)$ from the difference of $\mathbf{y}_{1}$ and $\mathbf{y}_{0}$. Consequently, the covariance of the position $\mathbf{x}\left(t_{0}\right)$ is equal to the covariance of the measurement error and the velocity covariance at time $t_{0}$ is $2 \Theta /\left(t_{1}-t_{0}\right)^{2}$.

\section{THE PARAMETER ESTIMATION METHOD}

The likelihood given by equation (17) depends on model parameters and measurements. Here we employ EM iterations for the parameter estimation [5]. In the E-step, we assume that $\sigma$ and $c$ are known and we estimate the force $\mathbf{f}_{0}$ using the Kalman filter. Symbolically expressed, it is given by

$$
E: \mathbf{f}_{0} \leftarrow \mathbf{K F}_{\mathbf{f}_{0}}\left(\sigma, c, \mathbf{y}_{1} \mathbf{y}_{2} \ldots \mathbf{y}_{N}\right)
$$

The values of $\sigma$ and $c$ are known either from the previous maximization M-step or they are initially guessed at the beginning of the iterations. The Kalman filter $\mathbf{K F}_{\mathbf{f}_{0}}$ is structurally the same as the one presented in the previous section. To accomplish the force estimation, its state vector is extended to include the force

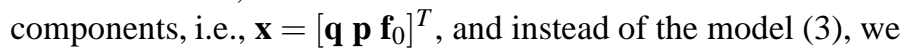
use

$$
\dot{\mathbf{x}}(t)=\mathbf{A}^{\mathbf{f}_{0}} \mathbf{x}(t)+\mathbf{L}^{\mathbf{f}_{0}} \xi(t),
$$

where

$$
\mathbf{A}^{\mathbf{f}_{\mathbf{0}}}=\left[\begin{array}{cc}
\mathbf{A} & \mathbf{0}_{2 D \times D} \\
\mathbf{0}_{D \times D} & \mathbf{I}_{D \times D}
\end{array}\right], \quad \mathbf{L}^{\mathbf{f}_{\mathbf{0}}}=\left[\begin{array}{c}
0_{D \times D} \\
\sigma \mathbf{I}_{D \times D} \\
0_{D \times D}
\end{array}\right]
$$


Consequently, in the Kalman filter equations (6)-(13), we use $\mathbf{A}=\mathbf{A}^{\mathbf{f}_{\mathbf{0}}}, \mathbf{L}=\mathbf{L}^{\mathbf{f}_{\mathbf{0}}}, \mathbf{B}=\mathbf{B}^{\mathbf{f}_{\mathbf{0}}}=0$ and $\mathbf{C}^{\mathbf{f}_{\mathbf{0}}}=\left[\mathbf{C} \mathbf{0}_{1 \times D}\right]$ and initialize the Kalman filter with

$$
\begin{gathered}
\mathbf{x}_{0}=\left[\begin{array}{c}
\mathbf{q}\left(t_{0}\right) \\
\mathbf{p}\left(t_{0}\right) \\
\mathbf{f}_{0}\left(t_{0}\right)
\end{array}\right]=\left[\begin{array}{c}
\mathbf{y}_{0} \\
\frac{1}{t_{1}-t_{0}}\left(\mathbf{y}_{1}-\mathbf{y}_{0}\right) \\
\mathbf{0}_{D \times 1}
\end{array}\right] \\
\mathbf{S}_{0}=\left[\begin{array}{ccc}
\Theta & \mathbf{0}_{D \times D} & \mathbf{0}_{D \times D} \\
\mathbf{0}_{D \times D} & \frac{2 \Theta}{\left(t_{1}-t_{0}\right)^{2}} & \mathbf{0}_{D \times D} \\
\mathbf{0}_{D \times D} & \mathbf{0}_{D \times D} & \Sigma_{F}
\end{array}\right]
\end{gathered}
$$

The force estimation is the $\hat{\mathbf{f}}_{0}\left(t_{N}\right)$ component of the Kalman filter state vector estimation $\left.\hat{\mathbf{x}}\left(t_{N}\right)=\left[\hat{\mathbf{q}}\left(t_{N}\right) \hat{\mathbf{p}}(t) N\right) \hat{\mathbf{f}}_{0}\left(t_{N}\right)\right]^{T}$. This estimation is optimal given the a priori information about the model and data [13].

In the M-step, we estimate $\sigma$ and $c$ maximizing the expectation of the likelihood (17) with respect to the distribution of the force estimation $\mathbf{f}_{0}$, which is assumed to be Gaussian with the mean value equal to the estimation $\hat{\mathbf{f}}_{0}\left(t_{N}\right)$ of the E-step and with the covariance $\Sigma_{F}$

$$
\begin{aligned}
& {\left[\begin{array}{c}
\hat{\sigma} \\
\hat{c}
\end{array}\right]=\arg \max _{\sigma, c} E_{\mathbf{f}_{\mathbf{0}} \sim N\left(\hat{\mathbf{f}}_{0}, \Sigma_{F}\right)}\left\{-\sum_{k=1}^{N} \mathbf{e}_{k} \mathbf{P}_{k}^{-1} \mathbf{e}_{k}+\right.} \\
& \left.\begin{array}{l|l}
\log \operatorname{det} \mathbf{P}_{k} & \mathbf{f}_{0}, \mathbf{y}_{\mathbf{k}}, k=0,1, \ldots
\end{array}\right\}
\end{aligned}
$$

The vertical bar $\mid$ implies that the expression inside the brackets (likelihood) is computed for given data and assuming that the force value $\mathbf{f}_{\mathbf{0}}$ is known exactly. The expression $\mathbf{f}_{\mathbf{0}} \sim N\left(\hat{\mathbf{f}}_{0}, \Sigma_{F}\right)$ in the subscript of the expectation operator denotes the expectation with respect to the distribution of $\mathbf{f}_{\mathbf{0}}$.

The covariance matrices $\mathbf{P}_{k}$ depend only on the parameters $\sigma, c$ and the initial covariance matrix $\mathbf{P}_{0}$. This means that we can remove the "log det" term from the expectation brackets and obtain:

$$
\begin{gathered}
{\left[\begin{array}{c}
\hat{\boldsymbol{\sigma}} \\
\hat{c}
\end{array}\right]=\arg \max _{\sigma, c}\left\{-\sum_{k=1}^{N} \log \operatorname{det} \mathbf{P}_{k}+\right.} \\
\left.\operatorname{tr}\left(E_{\mathbf{f}_{\mathbf{0}} \sim N\left(\hat{\mathbf{f}}_{0}, \Sigma_{\mathbf{f}_{0}}\right)}\left\{\mathbf{e}_{k} \mathbf{e}_{k}^{T} \mid \mathbf{f}_{0}, \mathbf{y}_{k}, k=0,1, \ldots\right\} \mathbf{P}_{k}^{-1}\right)\right\}
\end{gathered}
$$

Due to linearity of the model, the expectation can be rewritten as

$$
\begin{array}{r}
E_{\mathbf{f}_{\mathbf{0}} \sim N\left(\hat{\mathbf{f}}_{0}, \Sigma_{\mathbf{f}_{0}}\right)}\left\{\mathbf{e}_{k} \mathbf{e}_{k}^{T} \mid \mathbf{f}_{0}, \mathbf{y}_{\mathbf{k}}\right\}= \\
=\left.\mathbf{e}_{k} \mathbf{e}_{k}^{T}\right|_{\hat{\mathbf{f}}_{0}, \mathbf{y}_{\mathbf{k}}}+E_{\left(\mathbf{f}_{\mathbf{0}}-\hat{\mathbf{f}}_{0}\right) \sim N\left(0, \Sigma_{\mathbf{f}_{0}}\right)}\left\{\varepsilon_{k} \varepsilon_{k}^{T} \mid \mathbf{f}_{0}-\hat{\mathbf{f}}_{0}\right\} \\
=\left.\mathbf{e}_{k} \mathbf{e}_{k}^{T}\right|_{\hat{\mathbf{f}}_{0}, \mathbf{y}_{\mathbf{k}}}+\mathbf{P}_{k}^{\mathbf{f}_{\mathbf{0}}}
\end{array}
$$

with $\mathbf{P}_{k}^{\mathbf{f}_{\mathbf{0}}}$ being the covariance of the zero mean-value bias $\varepsilon_{k}$, at the time point $t_{k}$, and resulting from the assumption that the force $\mathbf{f}_{0}$ has a random value drawn from the Gaussian distribution. Because of that, the M-step can be written as

$$
\begin{array}{r}
{\left[\begin{array}{c}
\hat{\boldsymbol{\sigma}} \\
\hat{c}
\end{array}\right]=\arg \min _{\sigma, c}\left\{\left.\sum_{k=1}^{N} \mathbf{e}_{k} \mathbf{P}_{k}^{-1} \mathbf{e}_{k}\right|_{\hat{\mathbf{f}}_{0}, \mathbf{y}_{\mathbf{k}}}+\right.} \\
\left.\operatorname{tr}\left(\mathbf{P}_{k}^{\mathbf{f}_{\mathbf{0}}} \mathbf{P}_{k}^{-1}\right)+\log \operatorname{det} \mathbf{P}_{k}\right\}
\end{array}
$$

In the last expression, the minus sign in log-likelihood expression is removed and the maximization problem is substituted with the equivalent minimization problem of the negative log-likelihood.

To compute the covariance $\mathbf{P}_{k}^{\mathbf{f}_{\mathbf{0}}}$, we need to propagate the covariance $\Sigma_{F}$ to $\mathbf{P}_{k}^{\mathbf{f}_{\mathbf{0}}}$ through the Kalman filter in time. In the prediction step, the covariance propagation to the full state vector covariance $\Sigma_{k}^{\mathbf{f}_{\mathbf{0}}}$ can be computed as

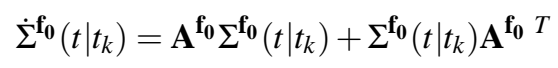

where

$$
\Sigma^{\mathbf{f}_{\mathbf{0}}}\left(t_{k} \mid t_{k}\right)=\mathbf{S}_{k}^{\mathbf{f}_{\mathbf{0}}}
$$

is the matrix A (see equation (3)) corresponding to Kalman filter $\mathbf{K F}_{\mathbf{f}_{\mathbf{0}}}$, and we start computing expression (29) with

$$
\mathbf{S}_{0}^{\mathbf{f}_{\mathbf{0}}}=\left[\begin{array}{ccc}
\mathbf{0}_{D \times D} & \mathbf{0}_{D \times D} & \mathbf{0}_{D \times D} \\
\mathbf{0}_{D \times D} & \mathbf{0}_{D \times D} & \mathbf{0}_{D \times D} \\
\mathbf{0}_{D \times D} & \mathbf{0}_{D \times D} & \Sigma_{F}
\end{array}\right]
$$

Expression (29) is similar to expression (7) in the prediction step of the Kalman filter, but the influence of the random process term is excluded. In the update step, we have

$$
\mathbf{S}_{k+1}^{\mathbf{f}_{\mathbf{0}}}=\left(\mathbf{I}-\mathbf{K}_{k}^{\mathbf{f}_{\mathbf{0}}} \mathbf{C}^{\mathbf{f}_{\mathbf{0}}}\right) \Sigma^{\mathbf{f}_{\mathbf{0}}}\left(t \mid t_{k}\right)\left(\mathbf{I}-\mathbf{K}_{k}^{\mathbf{f}_{\mathbf{0}}} \mathbf{C}^{\mathbf{f}_{\mathbf{0}}}\right)^{T}
$$

where $\mathbf{K}_{k}^{\mathbf{f}_{\mathbf{0}}}$ corresponds to the gain of the Kalman filter $\mathbf{K F}_{\mathbf{f}_{\mathbf{0}}}$. The latter equation results from the fact that we propagate only the influence of the force variation, so the measurement error term with $\Theta$ is also excluded. Finally, the covariance matrix $\mathbf{P}_{k}^{\mathbf{f}_{\mathbf{0}}}$ is

$$
\mathbf{P}_{k}^{\mathbf{f}_{\mathbf{0}}}=\mathbf{C}^{\mathbf{f}_{\mathbf{0}}} \mathbf{S}_{k}^{\mathbf{f}_{\mathbf{0}}} \mathbf{C}^{\mathbf{f}_{\mathbf{0}} T}
$$

In summary, E-step and M-step are described by expressions (20) and (28), respectively. To start iterations, we need to initially guess the values for $\hat{\sigma}, \hat{c}$ or $\hat{\mathbf{f}}_{0}$. In the case when it is not clear if 


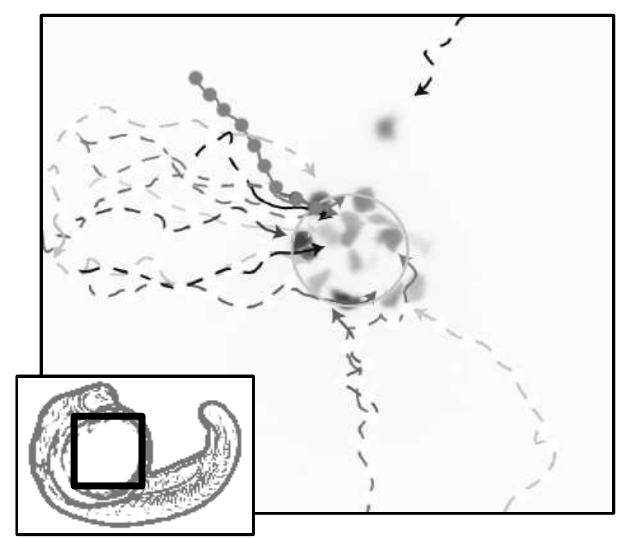

Figure 1. ZEBRAFISH EMBRYO DATA [1]: CELL TRACKS RESULTING FROM MIGRATION INDUCED BY THE LASER BEAM WOUND (DASHED); THE TRACK WE USE IN THE EXAMPLES (CIRCLES); CELLS ARE THE GRAY CLOUDY SHAPES. THE INSERTION SHOWS THE ZEBRAFISH EMBRYO AND THE SQUARE $323 \times 323 \mu m$ REGION IN WHICH THE CELLS ARE OBSERVED.

the force $\mathbf{f}_{0}$ actually exists, the initial guess of $\hat{\mathbf{f}}_{0}=0$ is justified. Moreover, we can compute the initial guess for $\hat{\sigma}$ and $\hat{c}$ using equation (25), in which case $\Sigma_{F}=\mathbf{0}$.

Starting from initial parameter values and force vector, EM iterations will result in the parameter values and force that lead to a higher value of the likelihood.

\section{ILLUSTRATIVE EXAMPLE}

One way to illustrate the proposed method is to estimate parameters and force from the data numerically generated based on the model given by equations (1) and (2) in which parameters and force are specified. While a close agreement between the estimated and the specified (the true values) will nicely validate the method, the level of the agreement cannot be warranted due to its dependence on the specified values and number of data points. In other words, if the close agreement is not reached, it does not invalidate the EM iterations of the method. Because of that, we illustrate the method using the real data. A more complex statistical analysis for computing the estimator confidence intervals is beyond the scope of this paper. In this way we not only illustrate the method, but also provide an example of its application.

We use the data obtained from the experiments with zebrafish embryo published in [1]. The experiments provide the data on migration of the immune system cells towards the wound produced by the laser beam. The inset in Fig. 1 presents zebrafish embryo with the square region in which the wound was produced. Figure 1 shows only one set of cell tracks from one of the experiments. This figure, as well as all the data we are dealing with here, are extracted from the supplementary material video "movie1" [1]. Exact description of the experiment is not essential for our example. For full details, please see the original paper [1].

Using the provided $50 \mu \mathrm{m}$ bar in video sequence, we can con- clude that the visual field, in which the cell tracks are recorded, is $323 \times 323 \mu \mathrm{m}$ and we used it to convert pixel coordinates of the cell center positions to $\mu \mathrm{m}$ distance measured from the leftbottom corner of the visual field. The exact time at which each frame is recored is not provided, but based on the sequence length and time record of the last frame $(60 \mathrm{~min})$, we concluded that the frames are sampled at the rate of 3 frames per minute $(20 s)$. The cell track we analyse in our example is marked by circles in Fig. 1 and plotted in Fig. 2. The corresponding data are provided in Table 1 at the end of this paper. Our measurements of the cell center positions are not error free. Our assumption is that the two-standard-deviation of the error along each axis is $1 / 4$ of an average observed cell radius $(8 \mu \mathrm{m})$, i.e., we assume the error covariance matrix $\Theta=\operatorname{diag}\{1,1\}$. The distance and time units that we use in this paper are $\mu m$ and $\min$, respectively, and all values and parameters are given in units corresponding to this spatio-temporal scale. To identify the parameters and the force, we first assumed $\hat{\mathbf{f}}_{0}=\left[\begin{array}{ll}f_{x} & f_{y}\end{array}\right]=\left[\begin{array}{ll}0 & 0\end{array}\right]^{T}$, and $\Sigma_{F}=\mathbf{0}$ and computed the initial guess for $\hat{\sigma}$ and $c$ using equation (25). The computed initial guess parameter values are $\hat{\sigma}=3.1087$ and $\hat{c}=0.1240$. Then we applied the EM iterations technique assuming $\Sigma_{F}=(2.5)^{2}$ and using initial values computed via equations (23) and (24). For the maximization for computing the initial guess, as well as in the M-step of EM iterations, we use the MATLAB function fmincon.

The EM iterations results are presented in Fig. 3. This figure shows that the negative log-likelihood converges towards the minimum. The EM iterations also lead to convergence of estimated values towards $\hat{\sigma}=7.7560, \hat{c}=2.5738$ and $\hat{\mathbf{f}}_{0}=$ $[17.8001-22.9089]^{T}$ as it is presented in Fig. 4 and Fig. 5. This validates numerically the EM approach presented in this paper. Finally, Fig. 2 shows that the estimated force direction is aligned with the cell trajectory in the image plane.

\section{CONCLUSIONS}

Following technological breakthrough in imaging, we are able to observe intravital cellular interactions. Estimating dynamical properties from video sequences of cellular processes is of importance for the development of experimental and diagnostic methods that minimally interfere with cell interactions. However, a computation estimation method that is based only on the visual information can suffer from convergence problems.

In this work, we have developed an expectationmaximization (EM) approach to estimate parameters and driving force of cell motility. The primary contribution of our work is in presenting these iterations for the case of continuous-time stochastic process describing cell motility. We illustrated the method using data from zebrafish embryo experiments. This example shows the type of data and results of estimation that can be obtained. Ultimately, this line of development leads to minimally-invasive experimental and diagnostic methods in biology and medicine.

Although we analyzed cell motility in this paper, in a simi- 


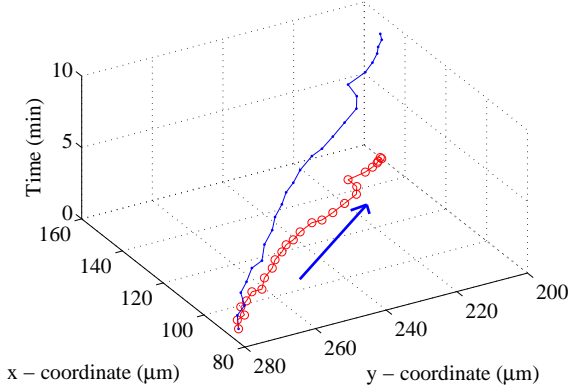

Figure 2. THE CELL TRACK IN RELATION TO THE ESTIMATION OF THE FORCE DRIVING CELL MOTILITY: CELL TRACK IN SPACE AND TIME (DOTS), CELL TRACK IN $x$-y COORDINATES OF THE IMAGE PLANE (CIRCLES), ESTIMATED FORCE DIRECTION IN THE IMAGE PLANE (ARROW)

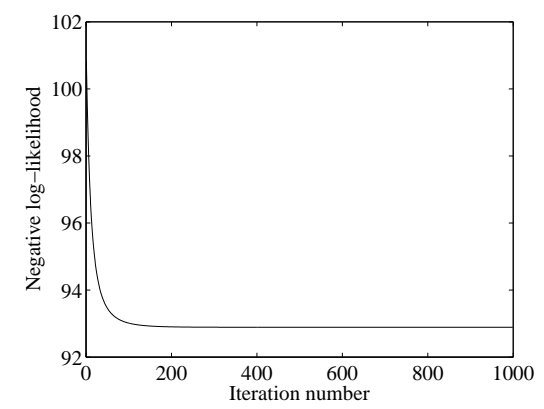

Figure 3. CHANGE OF NEGATIVE LOG-LIKELIHOOD OVER EM ITERATIONS
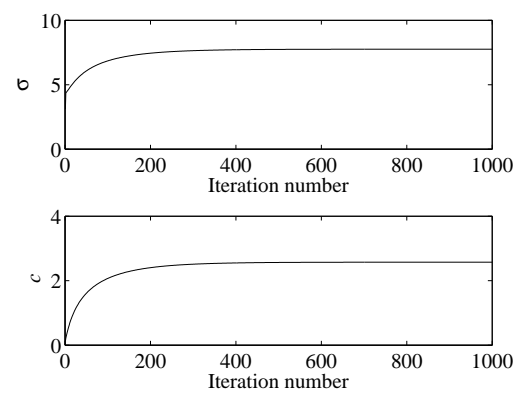

Figure 4. EM ITERATION-COMPUTED PARAMETER ESTIMATIONS
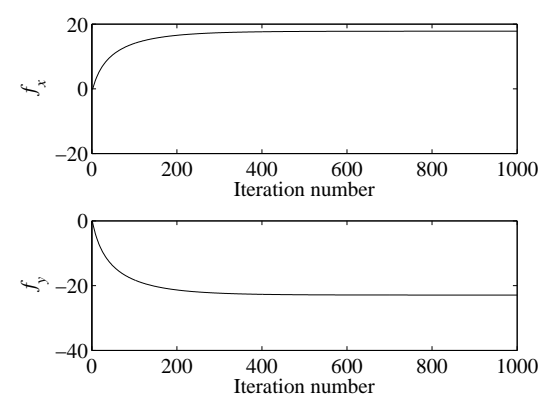

Figure 5. EM ITERATION-COMPUTED FORCE ESTIMATIONS, $\mathbf{f}_{0}=$ $\left[f_{x} f_{y}\right]^{T}$
Table 1. CELL-TRACK DATA

\begin{tabular}{|c|c|c|c|}
\hline$\#$ & Time $(\mathrm{min})$ & $\mathrm{x}(\mu \mathrm{m})$ & $\mathrm{y}(\mu \mathrm{m})$ \\
\hline 1 & 0.000 & 89.285714 & 276.428571 \\
2 & 0.333 & 93.571429 & 274.285714 \\
3 & 0.667 & 95.000000 & 271.428571 \\
4 & 1.000 & 99.285714 & 270.000000 \\
5 & 1.333 & 101.428571 & 267.142857 \\
6 & 1.667 & 105.000000 & 263.571429 \\
7 & 2.000 & 105.000000 & 260.714286 \\
8 & 2.333 & 110.000000 & 257.142857 \\
9 & 2.667 & 113.571429 & 252.857143 \\
10 & 3.000 & 117.142857 & 250.000000 \\
11 & 3.333 & 120.000000 & 246.428571 \\
12 & 3.667 & 122.857143 & 243.571429 \\
13 & 4.000 & 124.285714 & 240.714286 \\
14 & 4.333 & 127.142857 & 237.142857 \\
15 & 4.667 & 130.000000 & 232.142857 \\
16 & 5.000 & 130.000000 & 229.285714 \\
17 & 5.333 & 132.142857 & 225.000000 \\
18 & 5.667 & 135.000000 & 220.000000 \\
19 & 6.000 & 137.857143 & 215.000000 \\
20 & 6.333 & 141.428571 & 212.857143 \\
21 & 6.667 & 145.714286 & 212.857143 \\
22 & 7.000 & 147.142857 & 207.142857 \\
23 & 7.333 & 148.571429 & 204.285714 \\
24 & 7.667 & 150.000000 & 202.142857 \\
25 & 8.000 & 150.714286 & 201.428571 \\
26 & 8.333 & 151.428571 & 200.000000 \\
27 & 8.667 & 152.142857 & 200.000000 \\
\hline
\end{tabular}

lar fashion we can analyze video sequences of swimmers during a swimming race. In summary, there is a wide range of applications for estimating motility parameters and driving forces of moving particles, vehicles, insects, etc. in a non-invasive way, based upon video sequences.

\section{ACKNOWLEDGMENTS}

This research was supported by the Army Research Office under Short Term Innovative Research (STIR) grant number W911NF-07-R0003.

\section{REFERENCES}

[1] Redd, M. J., Kelly, G., Dunn, G., Way, M., and Martin, P., 2006. "Imaging macrophage chemotaxis in vivo: Studies of microtubule function in zebrafish wound inflammation". Cell Motility and the Cytoskeleton, 63, pp. 415-422.

[2] Miller, M. J., Wei, S. H., Parker, I., and Cahalan, M., 2003. "Autonomous t cell trafficking examined in Vivo with intravital two-photon microscopy". PNAS, 100, pp. 26042609.

[3] Mempel, T. R., Henrickson, S. E., and von Andrian, U. H., 
2004. "T-cell priming by dendritic cells in lymph nodes occurs in three distinct phases". Nature, 426, pp. 154-159.

[4] Milutinović, D., and Lima, P., 2007. Cells and Robots : Modeling and Control of Large-Size Agent Populations. Springer.

[5] Dempster, A. P., Laird, N. M., and Rubin, D. B., 1977. "Maximum likelihood from incomplete data via the EM algorithm". Journal of the Royal Statistical Society B, 39(1), pp. 1-38.

[6] Ding, F., and Chen, T., 2005. "Hierarchical identification of lifted state-space models for general dual-rate systems". IEEE Transactions on Circuits and Systems, 52(6), pp. 1179-1187.

[7] Ding, F., Qiu, L., and Chen, T., 2009. "Reconstruction of continuous-time systems from their non-uniformly sampled discrete-time systems". Automatica, 45(2), pp. 324-332.

[8] Digalakis, V., Rphlicek, J. R., and Ostendorf, M., 1993. "ML estimation of a stochastic linear system with the EM algorithm and its application to speech recognition". IEEE Transactions on Speech and Audio Processing, 1(4).

[9] Ljung, L., 1987. System Identification. Prentice-Hall.

[10] Kashyap, R. L., 1970. "Maximum likelihood identification of stochastic linear systems". IEEE Transactions on Automatic Control, AC-15(1), pp. 25-34.

[11] Gupta, N., and Mehra, R., 1974. "Computational aspects of maximum likelihood estimation and reduction in sensitivity function calculations". IEEE Transactions on Automatic Control, AC-19(6), pp. 25-34.

[12] Maine, R. E., and Iliff, K. W., 1981. "Formulation and implementation of a practical algorithm for parameter estimation with process and measurement noise". SIAM Journal on Applied Mathematics, 41(3), pp. 558-579.

[13] Gelb, A., 1974. Applied Optimal Estimation. M.I.T. press.

[14] Wu, C. F., 1983. "On the convergence properties of the EM algorithm". The Annals of Statistics, 11(1), pp. 95-103.

[15] Boyles, R. A., 1983. "On the convergence of the EM algorithm". Journal of the Royal Statistical Society B, 45(1), pp. $47-50$.

[16] Leonard, N., and Fiorelli, E., 2001. "Virtual leaders, artificial potentials and coordinated control of groups". In Proceedings of IEEE International Conference on Decision and Control, pp. 2968-2973.

[17] Tanner, H. G., Jadbabaie, A., and Pappas, G. J., 2007. "Flocking in fixed and switching networks". IEEE Transactions on Automatic Control, 52(5), pp. 863-868.

[18] Olfati-Saber, R., 2006. "Flocking for multi-agent dynamic systems: Algorithms and theory". IEEE Transactions on Automatic Control, 51(3), pp. 401-420.

[19] Kumar, M., Milutinović, D., and Garg, D., 2008. "Role of stochasticity in self-organization of robotic swarms". In Proceedings of American Control Conference.

[20] Schweppe, F. C., 1965. "Evaluation of likelihood functions for gaussian signals". IEEE Transactions of Information Theory, 11(1), pp. 61-70. 